\title{
EL USO DE LAS MATEMÁTICAS EN LA CIENCIA ECONÓMICA
}

\author{
ANTONIO ZANELLA* \\ ... se tiene que adaptar el método al objeto \\ de estudio, y no al revés. \\ Hans Mayer
}

En este breve ensayo discutiremos la posibilidad del uso de las matemáticas en las ciencias sociales y en particular en economía. El desarrollo de la ciencia económica, en nuestra opinión, ha sido sesgado desde el siglo XIX por el indebido uso de metodologías erróneas. No se ha reconocido la diferencia esencial entre el objeto de estudio de las ciencias naturales y el de las ciencias sociales y el resultado ha sido un monismo metodológico muy perjudicial para la ciencia económica. En este trabajo queremos mostrar las diferencias existentes entre los dos ámbitos de estudio y demostrar que el único enfoque posible y realmente científico es el dualismo metodológico.

I

\section{EL OBJETO DE ESTUDIO DE LA CIENCIA ECONÓMICA}

En este apartado intentaremos averiguar cuál es el objeto de estudio de las ciencias sociales y cuáles son sus características peculiares, para después monstrar cual es la metodología correcta para estudiarlo.

Las ciencias sociales, entre las cuales se encuentra la economía, estudian los seres humanos y sus interacciones que se plasman en la sociedad, entendida como una "complejísima red de

* Estudiante de Doctorado. Universidad Rey Juan Carlos. 
interacciones humanas» (Jesús Huerta de Soto); este hecho hace muy peculiar la relación entre el científico y el objeto de su estudio. En el caso de las ciencias sociales científico y objeto comparten la misma naturaleza, es decir los dos son hombres de carne y hueso. Este hecho es una gran ventaja a la hora de explicar y describir la realidad, pero al mismo tiempo es una gran desventaja cuando se trata de predecir acontecimientos futuros (criterio básico de cientificidad de las ciencias naturales basadas en la metodología positivista). Es una ventaja porque al compartir la misma naturaleza con su objeto, el científico sabe exactamente cuál es la causa última de todos los acontecimientos que estudia. Por otra parte es una desventaja porque el objeto que intenta estudiar es el más complejo que existe en el universo: el ser humano tiene una innata capacidad creativa para descubrir nuevos fines y medios y actuar en consecuencia para lograr sus fines utilizando esos medios; esta capacidad creativa es la razón esencial por la cual en economía no se pueden hacer predicciones cuantitativas. Veremos ahora las características peculiares del ser humano como objeto de estudio de la ciencia económica.

\section{Acción y movimiento}

El científico, por el hecho de compartir la misma naturaleza con el objeto que intenta estudiar, tiene la ventaja de saber de primera mano que es lo que lo «mueve», es decir puede conocer la causa última de todos los acontecimientos económicos. La diferencia fundamental entre las ciencias sociales y las naturales radica en la diferencia esencial que existe entre «movimiento» y «acción» (no toda acción implica movimiento y no todo movimiento implica acción). El concepto de movimiento puede verse como más amplio del de acción pero de ninguna manera puede ser mas preciso. El científico natural puede concebir el movimiento como la percepción de una alteración del espacio físico que lo rodea. El científico social puede percibir la acción aunque no haya alteración alguna del espacio físico, porque lo importante no es la manifestación física de la acción sino su significado para el actor en cuestión. Mises define la acción como «... una conducta 
consciente, movilizada voluntad transformada en actuación, que pretende alcanzar precisos fines y objetivos; ... una reacción consciente del ego ante los estímulos». ${ }^{1}$ Cuando un científico percibe un movimiento no sabe cuáles son las fuerzas originarias de tal modificación del espacio físico, lo que si puede notar es una correlación entre dos eventos particulares. El científico social, por su parte, al estudiar la sociedad conoce el dato último o sea la causa inicial de cada «movimiento»: la acción humana, y su fin. El científico social, en suma, conoce cuáles son las fuerzas que provocan el cambio en la sociedad.

\section{Causa o función}

La diferencia entre acción y movimiento nos lleva a la posibilidad de utilizar dos diferentes métodos de investigación, el método genético causal (y teleológico) y el método funcional. Si el objeto de estudio es el ser humano y sus acciones y como científicos compartimos su naturaleza o sea sabemos que es lo que significa actuar y cuáles son las causas ultimas de las acciones (los fines perseguidos por los individuos), entonces para explicar la realidad social el método mas adecuado será el método genético causal. Conocemos la causa última de los acontecimientos y entonces podremos explicar la realidad a partir de este dato último (ultimate given) que es la acción humana individual. En las ciencias naturales no tenemos la misma información, no sabemos cuáles son los datos últimos que causan el movimiento. Esto nos lleva al intentar explicar la realidad poniendo en relación dos o más elementos, pero sin saber cuál es el determinante y cual es el determinado. Esta ignorancia sobre la causa última se recoge en la expresión funcional, con la cual se intenta establecer una relación entre los elementos pero sin determinar una causalidad directa. En el campo de la acción humana, además, no existen constantes, todo son variables y este hecho hace que sea imposible la aplicación del método funcional en este ámbito.

${ }^{1}$ Ludwig von Mises, La Acción Humana, Unión Editorial, 9.․ edición, Madrid 2009, p. 15. 
La idea de la mutua determinación de los elementos, típica del enfoque funcional, es aplicable a la física, que quiere explicar movimiento de materia inconsciente; en la praxeología, al contrario, se conoce la causa ultima de todos los acontecimientos: la acción individual. $^{2}$

\section{Ideas, hechos y experimentos}

En el mundo de la acción humana no existen hechos empíricos observables de por si, sino solamente ideas que el científico tiene que comprender e interpretar. Todo «hecho» económico tiene que ser interpretado con las categorías propias de la acción humana (fin, medio, utilidad, tiempo, etc.); mediante la simple observación ningún acontecimiento social puede ser comprendido. Desde el punto de vista físico no se puede distinguir entre un papel blanco y un papel con la cara dibujada de uno de los presidentes de los Estados Unidos (excepto por la diferencia de color) pero desde el punto de vista de la teoría económica se distingue claramente entre un papel normal y el dinero. ${ }^{3}$ La diferencia entre el simple papel y el dinero depende de lo que creen y piensan subjetivamente los actores implicados en el proceso social. Un hecho muy sencillo como el intercambio entre dos individuos en que uno ofrece papel y el otro a cambio le da un coche no seria comprensible sin la aplicación de los conceptos praxeologicos (praxeología entendida como ciencia de la acción humana) de acción, medio, utilidad, dinero, etc. Además, siendo la economía una ciencia que estudia ideas, y no existiendo constantes sino solo variables, no es posible hacer experimentos de laboratorio como se hacen en las ciencias naturales. No se puede aislar y mantener constante solamente un hecho para ver como se comportan todos los demás.

\footnotetext{
2 Praxeología es la ciencia de la accion humana.

${ }^{3}$ Esta diferencia entre «dolar» y el papel no esta dada para simpre, algun día los individuos podrian dar el mismo valor a los dos, dependiendo de las politicas monetarias de la FED (Federal Reserve).
} 


\section{Distinción entre teoría e historia}

No existiendo hechos empíricos de los que inductivamente sacar teorías universales, en economía se necesita una teoría previa, a priori, para poder interpretar la realidad. Mises, hablando del uso de las estadísticas relacionadas con los precios dice: «... lo único que la experiencia nos dice y asimismo lo único que la estadística recoge, es determinado precio efectivamente pagado en determinado lugar y fecha por cierta cantidad determinada de mercancía. Formar grupos con tales precios, así como deducir promedios de los mismos, equivale a basarse en reflexiones teóricas, las cuales, lógica y temporalmente, anteceden a dichas operaciones». ${ }^{4}$ Mises distingue claramente entre teoría e historia; la teoría, sostiene Mises, no puede ser extraída de la experiencia histórica, porque la historia misma es un conjunto de hechos complejos que sólo se pueden comprender e interpretar con una teoría previa. La teoría económica tiene que ser apriorística y deductiva, o sea tiene que partir del axioma de la acción humana y deducir los teoremas económicos con el razonamiento lógico deductivo. El punto de partida de la teoría es el axioma de la acción humana, que todos los individuos reconocen con la introspección y no pueden negar sin caer en una evidente contradicción: el mero hecho de negar el axioma presupone acción.

\section{Lógica verbal frente a matemática}

A partir del axioma de la acción el científico deduce mediante el razonamiento lógico deductivo los teoremas económicos fundamentales; teoremas que son validos con certeza apodíctica si es que en el razonamiento no ha habido errores lógicos y los supuestos de partida son reales. Podríamos distinguir entre cuatro tipos de teoremas económicos: (i) teoremas universalmente validos y relevantes, porque no ha habido error alguno en la cadena de razonamientos lógicos deductivos y los supuestos de partida se

\footnotetext{
${ }^{4}$ Mises, op. cit., p. 67.
} 
dan en el mundo real que queremos explicar; (ii) teoremas universalmente validos e irrelevantes, porque a pesar de la rectitud de los razonamientos los supuestos insertados en la cadena lógico deductiva son irreales; (iii) teoremas incorrectos pero relevantes, o sea teoremas deducidos con razonamientos lógicos erróneos pero con supuestos reales; y finalmente (iv) teoremas incorrectos e irrelevantes, por haber sido deducidos con razonamientos lógicos incorrectos y a partir de supuestos irreales. La única manera para poder averiguar la certeza de los teoremas económicos es averiguar si los supuestos de partida son relevantes y si ha habido vicios lógicos en la cadena de razonamientos lógicos deductivos. Rothbard en su articulo «Toward a Reconstruction of Utility and Welfare Economics» sostiene que el uso de la lógica en economía es correcto porque el objeto de estudio son hombres que actúan lógicamente y además añade que la matemática puede ser útil en física, donde el objeto de estudio es muy diferente y los pasajes lógicos no son significativos. Los axiomas y las deducciones de la física no tienen significado, lo tienen solamente en un sentido operacional, o sea son útiles porque pueden explicar y predecir hechos. En praxeología, en cambio, los axiomas son ciertos y tienen significado de por sí; cada paso en la deducción es significativo y cierto. Además la matemática, respecto a la lógica, es un lenguaje preciso pero muy limitado, que no puede recoger toda la complejidad del mundo social y de los seres humanos que lo constituyen. El matemático Karl Menger, hijo de Carl Menger fundador de la Escuela Austriaca de Economía, decía que el lenguaje de la lógica verbal utilizado por su padre de ninguna manera era menos preciso que el lenguaje matemático, la única diferencia era que la lógica verbal permitía el tratamiento de hechos mucho mas complejos. Huerta de Soto añade que es absurdo encorsetar la economía en un lenguage tan limitado como el matemático, dado que la economía ya tiene un lenguage perfecto para representar el objeto de estudio que abarca: el lenguaje que utilizan los seres humanos a la hora de comunicarse, pensar o representar el mundo en el día a día. Este lenguaje es la lógica verbal que, lejos de ser impreciso, tiene una riqueza y una capacidad de representar la realidad económica infinitamente mayor que el lenguaje matemático. Este lenguaje ha sido refinado por miles y 
miles de años de evolucion, llegando a una capacidad de abstración y representación impensables para el lenguage matemático.

\section{Individualismo metodológico y agregados macroeconómicos}

Si el objeto de estudio de la ciencia económica es el ser humano, con sus características reales de creatividad empresarial, y el punto de partida de la investigación económica es el axioma de la acción humana, al cual se van añadiendo supuestos para poder deducir lógicamente leyes económicas universales (es decir validas en cualquier tiempo y lugar) y siempre más complejas, otra característica fundamental de la metodología económica es el individualismo metodológico: es decir, toda ley económica tiene que ser retrotraible a la acción humana individual. Se podría decir que el individualismo metodológico es un punto de partida de toda investigación económica y un test para averiguar la bondad de las leyes económicas deducidas por el científico. Para que una ley económica sea cierta, tiene que ser retrotraible a las acciones humanas individuales y cuando falta esta conexión entre leyes y acciones el científico tendría que volver a someter a prueba la cadena lógico deductiva. Muy diferente es el enfoque que tiene al respecto la economía matemática; sobretodo en su diferenciación entre micro y macroeconomía se pierde cualquier conexión entre seres humanos de carne y hueso y las variables macroeconómicas de los modelos. Desaparece el ser humano (que también en la microeconomía neoclásica permanece oculto detrás de funciones) para dejar sitio a variables macroeconómicas como el PIB (producto interior bruto), M (masa monetaria), o P (nivel general de precios). En este enfoque la disciplina económica se divide en dos ramas diferentes casi inconexas entre si y ninguna de las leyes macroeconómicas pueden ser retrotraídas al comportamiento de los individuos de carne y hueso y a sus acciones. Los agregados macroeconómicos esconden las realidades económicas subyacentes porque este es un sacrificio necesario para que los hechos económicos puedan ser tratados de manera matemática, con funciones, ecuaciones de equilibrio y gráficos. 
En los próximos apartados entraremos mas en detalle discutiendo otras diferencias fundamentales entre los dos enfoques e intentaremos averiguar cuáles de los supuestos sobre los que se basan estos dos enfoques se adaptan más al objeto de estudio proprio de la ciencia económica.

\section{II \\ ASUNCIÓN DE CONTINUIDAD}

El enfoque matemático se basa en la asunción de continuidad. La realidad, para que pueda ser tratada con métodos matemáticos, tiene que ser continua. Bryan Caplan en su trabajo «Why I'm Not an Austrian Economist» explica que una función para ser diferenciada, tiene que ser continua y además que sin el supuesto de continuidad no se pueden diseñar simples construcciones algebraicas como las curvas de oferta y demanda, porque si las diseñas como un conjunto de puntos discretos las curvas en general no se cruzaran en ningún punto. Caplan subraya la importancia del supuesto de continuidad y afirma que «si Mises y Rothbard tienen razón, el cálculo no puede ser usado en economía». La Escuela Austriaca no admite el tratamiento matemático de las realidades económicas y una de las motivaciones más importante es que el supuesto de continuidad es absolutamente irreal en el ámbito de la acción humana. Rothbard sostiene que: «human beings act on the basis of things that are relevant to their action. The human being cannot see the infinitely small step; it therefore has no meaning to him and no relevance to his action». ${ }^{5}$ Esta cita nos lleva de nuevo a la diferencia fundamental que existe entre movimiento y acción que hemos destacado en el primer párrafo. Rothbard sostiene que el supuesto de continuidad es valido en física porque ésta se ocupa de objetos inanimados que se mueven pero no actúan, entendiendo la acción como comportamiento intencional del individuo que persigue fines y cuando actúa tiene en cuenta unidades relevantes de bienes

\footnotetext{
5 «Toward a Reconstruction of Utility and Welfare Economics», www.mises.org.
} 
económicos (ley de la utilidad marginal). Queremos decir que el individuo al actuar no se enfrenta a objetos físicos infinitamente divisibles, sino que se enfrenta a objetos que subjetivamente piensa que pueden ser útiles para lograr sus fines, y no tiene en cuenta al actuar una realidad continua e infinitamente divisible; lo que de verdad considera al actuar es la unidad relevante en el contexto de la acción para conseguir el fin prefijado. Un ejemplo sacado de la teoría de los precios puede ser útil para entender mejor este concepto. Los precios derivan de las valoraciones subjetivas que los individuos dan a los objetos y del hecho de que los individuos intercambien esos objetos. Cuando un individuo intercambia una determinada cantidad de dinero por otra cantidad de harina, no considera la harina como un bien infinitamente divisible (aunque físicamente se pueda considerar divisible al infinito), sino que la valora en base al uso que quiere hacer con esa harina. Para una ama de casa la unidad relevante a la que atribuye un determinado valor subjetivo puede ser un kilogramo para hacer la tarta de cumpleaños de su hijo, mientras que para un panadero la unidad relevante es una caja de harina de 50 kilos para poder hacer frente a la demanda diaria de pan de sus clientes. La unidad relevante depende siempre del contexto de la acción en el que el individuo se ve implicado, y es la valoración subjetiva del actor la que determina el precio de la unidad relevante de bien económico. La economía, en suma, es la ciencia que estudia la acción humana que persigue fines y utiliza medios para alcanzarlos y «medio» es todo lo que el actor considera útil para conseguir su fin (o sea lo que el actor tiene en cuenta a la hora de actuar). La continuidad, dice Rothbard, presupone pasos infinitamente pequeños, pero la acción humana es siempre discreta.

III

\section{EL CONCEPTO DE INDIFERENCIA}

Otro concepto fundamental que diferencia el enfoque neoclásico y matemático del enfoque austriaco es el de indiferencia. Uno de los pilares de la economía neoclásica son las curvas de indiferencia 
que se basan en el supuesto de indiferencia: $\mathrm{U}(\mathrm{a})=\mathrm{U}(\mathrm{b})$. No queremos entrar en la importancia que tiene el concepto de indiferencia para la construcción de las curvas, nuestro objetivo es simplemente criticar la base de este supuesto absolutamente irreal en el campo de la acción humana. $\mathrm{U}(\mathrm{a})=\mathrm{U}(\mathrm{b})$ significa que un individuo es indiferente entre dos alternativas.

Este supuesto no puede ser útil como base del análisis económico porque la economía es la ciencia de la acción humana y la acción humana ni demuestra indiferencia, ni puede basarse en la indiferencia. Al actuar el individuo siempre indica una preferencia por un determinado curso de acción respecto a otro y lo importante en economía es siempre la acción, no los motivos que llevan a la acción (concepto de preferencia demostrada). Rothbard acusa de psicologismo a los que intentan basar la economía en el supuesto de indiferencia, porque sostiene que las clases de indiferencia pueden existir en alguna parte de la mente humana, pero no son relevantes en economía, entendida como ciencia de la acción humana. ${ }^{6}$ Hülsmann añade ( Economic Science and Neoclassicism», www.mises.org) que la indiferencia como la conocemos gracias a la introspección es un hecho que tiene que ser explicado y no puede explicar la acción humana. Los neoclásicos quieren explicar el comportamiento humano a partir de otros hechos observables o hechos psicológicos que se pueden conocer introspectivamente $y$, añade Hülsmann, se equivocan en presuponer que existen relaciones constantes entre las condiciones de la acción y la acción misma. El intento de los neoclásicos es explicar fenómenos observables, como el comportamiento humano, en términos de otros fenómenos observables (como las condiciones físicas de la acción) o de fenómenos psicológicos (como grados de satisfacción de las necesidades). Estos fenómenos sin embargo, sostiene Hülsmann, son independientes y tienen que ser estudiados de manera separada, mientras que la economía es una teoría de la acción y no una psicología aplicada: los economistas de la Escuela Austriaca explican la

6 Ademas, añade Rothbard, las curvas de indiferencia se trazan empiricamente con cuestionarios en los que la gente puede mentir o simplemente pueden no reflejar las valoraciones subjetivas reales de los individuos. 
manifestación realizada de la acción humana (comportamientos y pensamientos) en términos de los elementos no realizados de la misma acción y esto porque cada acción humana contiene relaciones entre aspectos realizados y no realizados de la misma, como los fines realizados y las alternativas perdidas (concepto de coste de oportunidad).

IV

FUNCIÓN DE UTILIDAD Y ESCALA VALORATIVA

Los economistas neoclásicos utilizan las funciones de utilidad (FU) para describir las preferencias de los individuos, mientras que los economistas austriacos utilizan el concepto de escala valorativa. Bryan Caplan (op. cit.) sostiene que entre los dos conceptos no hay diferencia sustancial porque los dos son ordinales y sirven solamente para ordenar las preferencias.

La FU neoclásica se puede expresar de esta manera:

$\mathrm{U}=\mathrm{a}^{*} \ln ($ cantidad de manzanas $)+(1-\mathrm{a})^{*} \ln ($ cantidad de peras $)$;

Mientras que la escala valorativa se expresa:

$\mathrm{EV}:\left\{1 .^{\mathrm{a}}\right.$ manzana; $2 .^{\mathrm{a}}$ manzana; $1 .^{\mathrm{a}}$ naranja; $3 .^{\mathrm{a}}$ manzana $\}$.

Si efectivamente las dos expresiones son iguales, parece innecesario expresar las preferencias individuales con la FU, inútilmente mas complicada. Además, el uso del logaritmo neperiano y del símbolo (+) parce implicar una especie de cardinalidad en esta expresión, o sea que sea posible la aplicación de operaciones matemáticas. En suma, si las dos son ordinales no hay motivo para utilizar la expresión más complicada y si la FU es cardinal, entonces es un concepto profundamente equivocado si se aplica al ámbito de la acción humana. Rothbard subraya que el concepto de utilidad cardinal es equivocado porque las magnitudes psicológicas no pueden ser medidas, dado que no son ni objetivas ni extensivas, y que la acción humana no demuestra nada medible, sino simplemente que una alternativa ha sido preferida 
a otra. ${ }^{7}$ A diferencia de la FU, la EV es ordinal y la distancia entre las preferencias no se puede medir de ninguna manera; de hecho, añade Rothbard, seria mejor clasificar las preferencias con letras $\{\mathrm{A} ; \mathrm{B}, \mathrm{C}$; etc... $\}$ que con números, para que no haya confusión. Rothbard atribuye la confusión entre ordinalidad y cardinalidad al hecho de que después de la revolución marginalista de Menger, Jevons y Walras, se ha confundido el concepto económico de «marginal» con el marginal del cálculo diferencial. En matemática el total de algo es el «integral» de cosas marginales y la utilidad total es el integral matemático de una serie de utilidades marginales. De esta confusión deriva el concepto de utilidad cardinal a la que se aplican las operaciones matemáticas.

Algunos economistas han abandonado el concepto de utilidad cardinal y lo han sustituido por las curvas de indiferencia para describir las preferencias individuales; en el párrafo anterior ya hemos criticado este enfoque.

\section{$\mathrm{V}$ \\ MEDICIÓN Y PREDICCIONES CUANTITATIVAS}

En el apartado anterior hemos visto que las magnitudes psicológicas no se pueden medir. Ahora veremos cuál es el problema de la medición en la ciencia económica.

Rothbard define la medición como: «univoca asignación de números que se pueden manejar con la aritmética» (op. cit.). Para poder medir es necesario definir o establecer una unidad fija y para definir la unidad la propiedad que se tiene que medir tiene que ser extensiva en el espacio para que haya un acuerdo objetivo sobre la unidad establecida. Los estados subjetivos, las magnitudes psicológicas, son intensivos y no son medibles ni se puede aplicar la aritmética. Por este motivo en economía no cabe hacer medición alguna. Se ha dicho, para sostener la tesis contraria, que se ha conseguido medir una sensación subjetiva como el calor con una ciencia objetiva como la termometría. Pero la termometría,

\footnotetext{
7 En el próximo apartado discutiremos el concepto de medición.
} 
subraya Rothbard, no mide las sensaciones subjetivas de calor, sino que supone una correlación aproximada entre la propiedad intensiva que se quiere medir y un evento objetivo extensivo, como la expansión del mercurio. En realidad la termometría no mide la sensación subjetiva de calor, simplemente es una medida de la longitud. ${ }^{8}$ En economía no es posible medición alguna, porque la economía es la ciencia de la acción humana y la acción humana no se puede explicar en términos de fenómenos psicológicos y aunque se pudiera, los fenómenos psicológicos son intensivos y no se pueden medir por no poder establecerse una unidad fija. Al no ser posible medición alguna no se pueden aplicar métodos matemáticos en economía y no son posibles predicciones cuantitativas. La economía es una ciencia cualitativa y las predicciones posibles en esta ciencia son solamente predicciones cualitativas o como F.A. Hayek las llamaba, «pattern prediccions».

\section{VI}

\section{CONCEPTO DE PROBABILIDAD}

Mises distinguía entre dos conceptos diferentes de probabilidad: la probabilidad de clase y la probabilidad de caso o evento único. Jesús Huerta de Soto en su libro Socialismo, cálculo económico y función empresarial (Unión Editorial, 4. e edición, Madrid 2010, p. 47), subraya cinco diferencias esenciales entre el mundo de la ciencia natural y el mundo de la acción humana a los que se aplican los dos diferentes conceptos de probabilidad:

1. En la probabilidad de clase se conoce o puede llegar a conocerse el comportamiento de la clase, pero no el comportamiento individual de sus elementos; mientras que en la probabilidad de caso o evento único no existe clase pero se conocen algunos factores que afectan el evento único y otros no, la propia acción provoca, o crea, dicho evento.

8 Muchas veces, a pesar de que la temperatura sea igual, individuos diferentes (o el mismo individuo en momentos diferentes del tiempo) tienen sensaciones subjetivas diferentes de frío y calor. 
2. En el mundo de la ciencia natural existe una situación de riesgo asegurable por toda la clase; mientras que en el mundo de la acción humana hay incertidumbre inerradicable, dado el carácter creativo de la acción humana. La incertidumbre no es, por tanto, asegurable.

3. La probabilidad de clase es matematizable mientras que la probabilidad de caso no.

4. Se llega a la probabilidad de clase mediante la lógica o la investigación empírica y el teorema de Bayes permite aproximar la probabilidad de clase, conforme aparece nueva información. La probabilidad de caso se descubre mediante la comprensión y estimación empresarial. Cada nueva información modifica ex novo todo el mapa de creencias y expectativas (concepto de sorpresa).

5. La probabilidad de clase es objeto de investigación del científico natural, mientras que el concepto de probabilidad de caso es utilizado por el actor-empresario. O por el historiador.

Rothbard añade que Richard von Mises, hermano de Ludwig, ha demostrado que la probabilidad numérica puede ser aplicada solamente a situaciones donde hay una clase de entidades tal que nada se sabe sobre las entidades que la componen excepto que son miembros de esta clase y donde pruebas sucesivas revelan una tendencia asintótica hacia una proporción estable o frecuencia de ocurrencia de un determinado evento en esa clase. En el mundo de la acción humana, en cambio, no existen clases compuestas por miembros homogéneos, sino que existen solamente eventos únicos, no repetibles a los que no se puede aplicar el concepto de probabilidad numérica. El hombre que actúa se enfrenta a un «riesgo» no cuantificable (o incertidumbre inerradicable) porque la creación de nuevas ideas cambia continuamente la manera en que los seres humanos actúan bajo condiciones que sin estas nuevas ideas serian las mismas (Hülsmann, op. cit., p. 12). Hoppe (1989, p. 112; 1993, capítulo 7 y 1995, p. 36) por su parte explica que cada teoría determinística de la acción (como la probabilística) implica una insoluble contradicción: esta teoría tiene que presuponer relaciones constantes entre la acción (evento explicado estocásticamente) y sus condiciones (distribución 
estocástica); lo que presupone que el hombre no puede aprender porque cada idea nueva cambia la manera de actuar bajo las mismas circunstancias. Pero que el hombre no pueda aprender contradice el supuesto necesario de cualquier programa científico: que este programa pueda tener algún significado o sea que el hombre pueda aprender de el.

\section{VII \\ OTROS SUPUESTOS IRREALES DE LA ECONOMÍA MATEMÁTICA Y SUS CONTRADICCIONES}

En este párrafo, siguiendo el articulo de Hans Mayer «Il concetto di equilibrio nella teoría economica» (Economia Pura, Editrice Torinese, Torino 1937), analizaremos otros supuestos en los que se basa la economía matemática y del equilibrio que consideramos inaplicables al mundo de la acción humana.

\section{Simultaneidad}

En la economía matemática se supone que hay perfecta simultaneidad entre los elementos del sistema: cantidades demandadas y ofertadas, precios de bienes de consumo y de orden superior son elementos que ya existen simultáneamente desde el principio. El sistema que constituye la economía de mercado, según este enfoque, consiste en las relaciones cuantitativas entre estos elementos. Como hemos visto en los párrafos anteriores, en las ciencias sociales, a diferencia de las ciencias naturales, conocemos la causa última de los acontecimientos: la acción humana individual. Esto indica que no hay simultaneidad entre los elementos: existe una causa, que es la acción humana individual, y sus consecuencias o efectos. Este supuesto de simultaneidad es incompatible con el concepto de causalidad, que es la base de cualquier investigación económica. 


\section{Interdependencia}

Los elementos del sistema económico se consideran puntos materiales, con existencia independiente, puestos en movimiento unos hacia los otros por parte de fuerzas (misteriosas) hasta llegar al equilibrio. El concepto de interdependencia en mecánica racional deriva del hecho de que la experiencia física nos dice que los elementos se encuentran en determinadas relaciones entre si, relaciones que se pueden expresar con ecuaciones funcionales (en los párrafos anteriores ya hemos visto la diferencia entre movimiento físico y acción humana y la imposibilidad de usar las ecuaciones funcionales para expresar la realidad económica). Según este supuesto todos los elementos influyen sobre todos los otros. Este supuesto también, como la simultaneidad, implica la eliminación del concepto de causa en la ciencia económica. Si todos los elementos son interdependientes entonces no existen causas y efectos.

\section{Reversibilidad}

Es indiferente, según este enfoque, saber cuáles son las variables dependientes y cuales las independientes. En el tratamiento funcional se puede decir que $y=f(x)$ o que $x=g(y)$. No se sabe, en este caso, si es y la que determina $x$ o es $x$ la que determina $y$; la expresión funcional simplemente recoge una relación entre estos dos elementos. Cournot, por ejemplo, recoge su ley de la demanda en la ecuación:

$$
D=f(p)
$$

Esta ecuación presupone que los precios ya existen y que la cantidad demandada es función del precio. Pero se podría reescribir la expresión al revés y decir que $\mathrm{p}=\mathrm{g}(\mathrm{D})$, o sea que los precios, como variables dependientes existen en función de las cantidades demandadas, que están dadas y son las variables independientes. El enfoque genético-causal se enfrenta a la ley de la determinación de los precios de manera completamente diferente. El punto de partida es siempre la acción humana individual y sus valoraciones 
subjetivas ordinales como causa de todo acontecimiento económico. De la acción humana individual se deduce la teoría del valor subjetivo que, junto con la cataláctica (teoría del intercambio) y la teoría del dinero, explica de manera causal la formación de los precios. La teoría de los precios de la Escuela Austriaca de Economía es muy diferente de la neoclásica porque a través de la metodología genetico-causal deduce la ley de la formación de los precios directamente de la acción humana individual (deducción que satisface el supuesto del individualismo metodológico) y con esta teoría se entiende perfectamente que son las valoraciones subjetivas de los individuos (en concreto la valoraciones subjetivas de las parejas marginales) las que, encontrándose entre si a través del intercambio, determinan los precios de los bienes económicos; hay una clara distinción entre determinante y determinado y no una simple relación funcional en la que no se entiende cuál es el elemento que determina al otro. Mayer (op. cit., p. 668), además, añade que si desaparecieran los precios porque por ejemplo los individuos durante la noche los olvidaran, desaparecerían al mismo tiempo las demandas efectivas y entonces la teoría funcional de los precios neoclásica no seria capaz de deducir de ninguna manera nuevos precios. La teoría genetico-causal de los precios, al contrario, podría siempre deducir nuevos precios, porque las relaciones fundamentales que los forman, o sea las valoraciones subjetivas individuales, y las cantidades que cada actor posee, seguirían existiendo y a través del intercambio libre se formarían nuevos precios. Esta es la diferencia fundamental entre las teorías funcionales y las genético causales: las funcionales admiten que todos los elementos del sistema existan simultáneamente y se adapten recíprocamente los unos a los otros, mientras que en la teoría genetico-causal de la Escuela Austriaca algunos elementos son considerados como primarios y otros como derivados de estos.

\section{Tiempo, previsión y ser humano}

Al existir completa simultaneidad entre los elementos del sistema, no solamente no puede existir el concepto de causa sino que además tampoco puede existir el verdadero concepto (no 
«espacializado») de tiempo. La simultaneidad implica atemporalidad y la atemporalidad implica la imposibilidad de que exista una causa, que es por definición temporalmente anterior a su efecto. Si en la economía matemática del equilibrio no tiene sentido hablar ni de tiempo, ni de causa, tampoco tiene sentido hablar de previsión, esto porque el concepto de previsión implica un presente y un futuro que tiene que ser previsto. Al no poder hablarse de previsión, característica esencial del ser humano, no hay hombre que actúa en este sistema económico y sin hombre se quita del medio el mismo objeto que esta ciencia pretendía estudiar; la economía matemática cae en una contradicción insoluble. Sin tiempo, causalidad, previsión etc., no hay acción humana, porque la acción presupone el pensar en el presente que actuando se puede modificar (concepto de causa y efecto) el futuro. La misma expresión «economía del equilibrio» es una contradicción en los términos, porque economía es la ciencia de la acción humana mientras que el equilibrio presupone los conceptos de simultaneidad, atemporalidad y reversibilidad que implican la imposibilidad (o ausencia) de cualquiera acción humana.

Sucesivamente las teorías matemáticas han intentado incluir el concepto de tiempo en sus construcciones como una sucesión de momentos idénticos entre si $\left(t_{1}, t_{2}, t_{3}, \ldots.\right)$; lo que ha creado la ilusión que se podía incluir el tiempo en estos sistemas y evitando supuestos tan irreales como los de simultaneidad etc. En realidad, el concepto de tiempo proprio de la física es muy diferente del concepto de tiempo proprio de la acción humana. El tiempo en física es simplemente una sucesión de momentos iguales entre si que llevan a un futuro «dado» o «por venir» (Huerta de Soto 2010, cap. II), mientras que el tiempo de la acción humana es un tiempo subjetivo o sea un tiempo cuyo transcurso es percibido por el actor conforme actúa en el correspondiente contexto de la acción. El tiempo de la economía es un tiempo en el sentido Bergsoniano del término, que el actor percibe a nivel subjetivo mientras actúa y este concepto de tiempo no se puede matematizar con simples ( $\mathrm{t}$ ) todas iguales unas a las otras. El futuro, en economía es un «por hacer» (ibidem), o sea que no esta dado sino que tiene que ser creado por las acciones de los seres humanos. Además, el concepto praxeológico de tiempo no permite tratamiento matemático y lleva a una 
definición muy diferente de los conceptos de pasado, presente y futuro. El presente es todo lo que el individuo considera como tal en el contexto de la acción que está llevando al cabo, su duración no es predeterminada sino que varia según la acción emprendida por el actor.

Queremos, concluyendo, subrayar que sin el concepto de tiempo no se pueden comprender hechos económicos fundamentales como la preferencia temporal, el tipo de interés (entendido como el precio de los bienes futuros en relación con los presentes), el dinero (entendido como todo medio de intercambio común y generalmente aceptado, cuya función fundamental es su capacidad de hacer frente a la incertidumbre futura), las expectativas, la estructura productiva entendida como estructura por etapas, etc.

\section{VIII \\ CONTRADICCIONES INSOLUBLES \\ EN LAS TEORÍAS DEL EQUILIBRIO}

Hans Mayer en su artículo destaca tres características principales de las teorías del equilibrio:

1. Entienden la economía como el estudio de las relaciones cuantitativas entre los elementos.

2. Consideran que el objetivo de la economía es la determinación de las ecuaciones funcionales de equilibrio de los elementos.

3. Utilizan ecuaciones diferenciales para llegar a una determinación univoca del equilibrio.

Mayer subraya que las teorías matemáticas del equilibrio tienen algunas contradicciones importantes. La primera es que estas teorías pretenden ser ciencias experimentales que, sin embargo, parten de generalizaciones inadmisibles (como la interdependencia general entre los elementos) e hipótesis irreales sobre los elementos. Además, estudian una situación irreal e inalcanzable de equilibrio donde el hombre, que supuestamente tendría que ser el protagonista de toda análisis económica, brilla por su ausencia. Estas teorías, por otro lado, no llegan a una solución univoca de 
las ecuaciones diferenciales a pesar de que el fin inicial era demostrar la unicidad de determinadas relaciones. Esto, según Mayer, puede significar que el planteamiento del problema no es adecuado al objeto de estudio y añade que se tiene que adaptar el método al objeto de estudio y no al revés.

El concepto de equilibrio puede entenderse de dos maneras. El equilibrio se puede considerar como un estado de reposo, y esto supone la inexistencia de toda acción humana o sea lo contrario de lo que se entiende por economía. En cambio, se puede entender el equilibrio como equilibrio en movimiento: no existen variaciones de los elementos, unos respecto de otros, pero el sistema en su totalidad sigue en movimiento. En este segundo caso tenemos que preguntarnos cuáles son las fuerzas que mantienen en movimiento el sistema. Esta fuerza puede ser endógena (como por ejemplo la renovación permanente de las necesidades) y, en este caso, cae la interdependencia de todos los factores porque existe un elemento activo y no determinado, una causa y esto supondría una contradicción con la interdependencia postulada en el modelo; o bien esta fuerza puede ser exógena y, en este caso, sostiene Mayer, la descripción del equilibrio seria incompleta faltando el elemento que mantiene viva a la economía: el sistema no seria cerrado y de nuevo no habría interdependencia entre los elementos sino una causa externa. Mayer compara este sistema a un reloj en que todos los elementos se mueven gracias a un impulso exterior, por ejemplo una persona que le da cuerda cada periodo de tiempo.

Como hemos visto en este apartado las teorías matemáticas del equilibrio caen en evidentes e insolubles contradicciones. En el próximo apartado analizaremos brevemente el enfoque de la Escuela Austriaca.

\section{IX \\ EL PARADIGMA DE INVESTIGACIÓN DE LA ESCUELA AUSTRIACA}

En los anteriores apartados hemos visto cómo los supuestos sobre los que se basan las teorías económicas del equilibrio que usan la metodología matemática son profundamente irreales y 
llevan a conclusiones contradictorias. El enfoque de la Escuela Austriaca de Economía es muy diferente y no estamos de acuerdo con Bryan Caplan cuando en su articulo dice que entre las dos escuelas no hay suficientes diferencias como para que se puedan considerar dos paradigmas distintos. En nuestra opinión los dos enfoques no solamente son tan diferentes que es correcto hablar de paradigmas opuestos, sino que además sus supuestos, metodologías y conclusiones son incompatibles y no pueden confluir en un mismo paradigma. Para concluir este breve ensayo queremos presentar una breve visión global del proyecto de investigación de la Escuela Austriaca, para subrayar sus profundas diferencias respeto al enfoque propio de la economía matemática.

La Escuela Austriaca de Economía define la economía como la parte mas desarrollada de la praxeologia, entendida esta última como ciencia de la acción humana (Mises, La Acción Humana). Siendo la economía ciencia de la acción humana su objeto de estudio es el hombre real de carne y hueso y la sociedad, definida como complejísima red de interacciones humanas (Huerta de Soto, Socialismo, cálculo económico y función empresarial, fin del cap. II). El punto de partida, entonces, es el ser humano, pero no una simple caricatura sino un ser humano real analizado sin dejar de lado sus características principales y en especial su innata capacidad creativa (lo que Israel Kirzner y Jesús Huerta de Soto llaman «empresarialidad»). El postulado de partida es el axioma de la acción humana, o sea que el hombre actúa (y actúa para pasar de una situación de menor a una situación de mayor satisfacción); éste es considerado un axioma en el sentido que nadie lo puede negar sin contradecirse a si mismo (negar que el hombre actúa presupone una acción). La misma introspección nos permite darnos cuenta y entender este axioma y sus categorías principales: fin, medio, valor, utilidad, tiempo, causalidad etc. El análisis de la Escuela Austriaca, además, no hace abstracción de conceptos fundamentales como los de tiempo y causalidad. Para entender fenómenos complejos como la sociedad, los economistas austriacos no intentan hacer supuestos irreales para que el objeto de estudio (el ser humano) encaje en una metodología matemática que le es ajena, sino que suponen condiciones simples en 
las que se encuentra el objeto de estudio y sucesivamente van añadiendo complicaciones (supuestos) que hacen el análisis mas cercano a la realidad que nos rodea: el programa de investigación empieza con el intercambio aislado o intrapersonal. El punto de partida es puramente formal y no tiene contacto con la experiencia empírica (axioma de la acción humana que se descubre introspectivamete y sus categorías); a continuación este enfoque supone un hombre real de carne y hueso en el mundo real que persigue fines y tiene medios escasos para perseguir estos fines (Economía de Robinson Crusoe). En esta situación de intercambio aislado derivan la teoría subjetiva del valor (que se basa en los conceptos de fin, valor, medio, utilidad y escala valorativa) que es la relación fundamental sobre la que se basa todo el siguiente desarrollo de la teoría económica; teoría del valor entendida como la fuerza originaria que pone en movimiento todo el proceso social. A partir de las condiciones simples de una economía cerrada, como hemos visto, se deducen las relaciones fundamentales comunes a todas las economías. Sucesivamente añaden otro elemento más para que el análisis vaya acercándose a la compleja realidad social que quiere explicar: «Viernes». $\mathrm{El}$ intercambio de aislado pasa a ser interpersonal (intercambios entre dos seres humanos, Robinson Crusoe y Viernes) para después ampliar el análisis al proceso social de mercado. A partir de estos supuestos más complejos, deducen la teoría de los precios, la teoría del dinero (pasando de una economía de trueque o intercambio directo al intercambio indirecto con el uso del dinero definido como medio de intercambio común y generalmente aceptado), la teoría del capital, la teoría del ciclo económico y la teoría de los diferentes tipos de mercado (economía de mercado libre, economía socialista y economía intervenida).

En suma, a partir de supuestos reales y simples con el método genético causal y lógico deductivo, añadiendo condiciones siempre más complejas, deducen todo el conjunto de teorías económicas; teorías que respetan el principio fundamental del individualismo metodológico: cada ley económica tiene que ser retrotraible a la acción humana individual. 


\section{REFERENCIAS BIBLIOGRÁFICAS}

CAPLAN, B. (2003): «Why I'm Not an Austrian Economist», http:/ / www.gmu.edu/depts/economics/bcaplan/whyaust.htm

Hoppe, H.H. (1989): A Theory of Capitalism and Socialism, Kluwer, Boston.

- (1993): The Economics and Ethics of Private Property, Kluwer, Boston.

- (1995): Economic Science and the Austrian Method, Mises Institute, Auburn.

HÜLSMANN, J.G. (1999): «Economic Science and Neoclassicism», The Quarterly Journal of Austrian Economics, Vol. 2 (4): 3-20.

KIRZNER, I. (1998): Competencia y empresarialidad, Unión Editorial, Madrid.

MAYER, H. (1937): «Il concetto di equilibrio nella teoria economica», in Economia Pura, Editrice Torinese, Torino.

Mises, L. von (2009): La Acción Humana, 9. a ed., Unión Editorial, Madrid.

RothbARD, M.N. (1956): «Toward a Reconstruction of Utility and Welare Economics», http: / / mises.org/rothbard/toward.pdf

Huerta De SOTO, J. (2010): Socialismo, cálculo económico y función empresarial, Unión Editorial, 4. ${ }^{\mathrm{a}}$ ed., Madrid, (1. ${ }^{\mathrm{a}}$ ed. de 1992). 